\title{
The Hellenistic Dynasties
}

All dates are BCE.

\author{
KINGS OF MACEDONIA
}

Argead Dynasty

$\begin{array}{ll}\text { 4I3-399 } & \text { Archelaos } \\ 399-397 & \text { Orestes } \\ 397-394 & \text { Aëropos II } \\ 394-393 & \text { Amyntas II } \\ 394-393 & \text { Pausanias (rival king) } \\ 393-385 & \text { Amyntas III } \\ 385-383 & \text { Argaios II } \\ 383-370 & \text { Amyntas III (restored) } \\ 370-368 & \text { Alexander II } \\ 368-365 & \text { Ptolemy of Aloros (regent) } \\ 365-359 & \text { Perdikkas III } \\ 359-336 & \text { Philip II } \\ 336-323 & \text { Alexander III the Great } \\ 323-3 \text { I } & \text { Philip III Arrhidaios } \\ 323-309 & \text { Alexander IV }\end{array}$

Antipatrid Dynasty

305-297 Kassandros

297 Philippos IV

297-294 Alexander V

297-294 Antipatros I

\section{Civil Wars}

294-287 Demetrios I Poliorketes

287-285 Pyrrhos of Epeiros

287-28 I Lysimachos

28I-279 Ptolemy Keraunos 
279 Meleagros

279 Antipatros II 'Etesias'

279-277 Sosthenes (stratēgos only)

277-274 Antigonos II Gonatas

274-272 Pyrrhos of Epeiros, again

272-239 Antigonos II (restored)

THE ANTIGONID DYNASTY

306-30I Antigonos I Monophthalmos

306-287 Demetrios I Poliorketes

287-239 Antigonos II Gonatas

239-229 Demetrios II

229-22I Antigonos III Doson

22I-I79 Philip V

I79-I68 Perseus

THE ATTALID DYNASTY

283-263 Philetairos (dynast only)

263-24I Eumenes I (dynast only)

24I-I97 Attalos I Soter (king in 238/7)

I97-I59 Eumenes II Philadelphos

I 59-I 38 Attalos II Philadelphos

I38-I33 Attalos III Philometor

THE SELEUKID DYNASTY

3 I 2-28 I Seleukos I Nikator

28I-26I Antiochos I Soter

26I-246 Antiochos II Theos

246-226 Seleukos II Kallinikos

226-222 Seleukos III Soter

223/2-I 87 Antiochos III the Great

I 87-I75 Seleukos IV Philopator

I75-I64 Antiochos IV Epiphanes

I64-I62 Antiochos V Eupator

I62-I 50 Demetrios I Soter

I 50-I 45 Alexandros I Balas

I45-I42 Antiochos VI Epiphanes

I45-I39 Demetrios II Nikator

I39-I 29 Antiochos VII Sidetes 
I 29-I 25 Demetrios II (restored)

I 28-I 22 Alexandros II Zabinas

I 25-I20 Kleopatra I Thea

$\mathrm{I} 25$ Seleukos V

I 25-96 Antiochos VIII Grypos

I $3-95$

96-95 Antiochos IX Kyzenikos

$95-88$ Seleukos VI Epiphanes

$95-88$

95

$95-83$

$86-85$

$83-69$

$85-58$

$69-64$

66-63

Demetrios III Eukairos

Antiochos X Eusebes

Antiochos XI Epiphanes

Philip I Epiphanes

Antiochos XII Dionysos

Kleopatra II Selene (regent)

Seleukos VII Philometor?

Antiochos XIII Asiatikos

Philip II Philorhomaios

THE PTOLEMAIC DYNASTY

323-282 Ptolemy I Soter (king 305)

282-246 Ptolemy II Philadelphos

246-222 Ptolemy III Euergetes

222-204 Ptolemy IV Philopator

204-I $80 \quad$ Ptolemy V Epiphanes

I 80-I45 Ptolemy VI Philometor

I70-I63 Ptolemy VIII Euergetes

I45-I 44 Ptolemy VII Philopator

I 45-I 6 Ptolemy VIII (restored)

I I6-IOI Kleopatra III Euergetes

I 6-107 Ptolemy IX Philometor

I07-88 Ptolemy X Alexandros

88-80 Ptolemy IX (restored)

80

Kleopatra Berenike

80

Ptolemy XI Alexandros

$80-5 \mathrm{I}$

$58-55$

Ptolemy XII Neos Dionysos

$5 \mathrm{I}-47$

Berenike IV

$5 \mathrm{I}-30$

$48-47$

Ptolemy XIII Philopator

$47-44$

Kleopatra VII Philopator

Arsinoë

$36-30$

Ptolemy XIV Philopator

Ptolemy XV Caesar ('Caesarion') 